\title{
Application of Diffusion-Weighted Imaging in the Detection of Active Sacroiliitis and the Comparison of Apparent Diffusion Coefficient and Relative Apparent Diffusion Coefficient Values
}

\author{
İclal Erdem TOSLAK, ${ }^{1}$ Meral BİLGİLİSOY FİLİZ, ${ }^{2}$ Bülent ÇEKİÇ, ${ }^{1}$ Ayşe Eda PARLAK, ${ }^{1}$ \\ Hasan Fatih ÇAY, ${ }^{3}$ Sevim YILDIZ, ${ }^{1}$ Bekir EROL, ${ }^{1}$ Deniz ÖZEL, ${ }^{4}$ İlhan SEZER ${ }^{3}$ \\ ${ }^{1}$ Department of Radiology, Antalya Training and Research Hospital, Antalya, Turkey \\ ${ }^{2}$ Department of Physical Therapy and Rehabilitation, Antalya Training and Research Hospital, Antalya, Turkey \\ ${ }^{3}$ Department of Rheumatology, Antalya Training and Research Hospital, Antalya, Turkey \\ ${ }^{4}$ Department of Biostatistics and Medical Informatics, Medical Faculty of Akdeniz University, Antalya, Turkey
}

\begin{abstract}
Objectives: This study aims to evaluate the role of diffusion-weighted imaging in detection of active sacroiliitis and compare the apparent diffusion coefficient $(A D C)$ and normalized relative $A D C$ ( $r-A D C)$ values by using vertebra and iliac wings as reference organs.

Patients and methods: The study included 56 patients ( 26 males, 30 females; mean age $37.7 \pm 10.1$ years; range 18 to 66 years) with chronic back pain and without history of sacroiliitis who underwent magnetic resonance imaging. $T_{2}$-weighted spectral presaturation with inversion recovery, contrast-enhanced $\mathrm{T}_{1}$-weighted spectral presaturation with inversion recovery, and diffusion-weighted (b values: 0 and $600 \mathrm{~s} / \mathrm{mm}^{2}$ ) images were obtained. All images were evaluated by two different radiologists for interobserver variability. All individuals were grouped in either mechanical low back pain (control group) or active sacroilitis (disease group) groups according to the presence or absence of MRI findings of active sacroilitis. $A D C$ values of both surfaces were measured from normal and affected areas of joints. Also, $A D C$ values of $L_{5}$ vertebra and iliac wings were measured as reference organs to calculate $r-A D C$ values.

Results: Mean $A D C$ and $r-A D C$ values measured from lesions were significantly higher than that of normal appearing bone marrow areas in both patients with mechanical low back pain $(n=17)$ and active sacroiliitis $(n=39)$. Both $A D C$ values and $r-A D C$ values could differentiate active lesions from normal appearing bone marrow areas as well as contrast-enhanced $T_{1}$-weighted images. According to $r-A D C$ values calculated with the $L_{5}$ vertebra, unaffected portions of bone marrow areas in patients with sacroiliitis were normalized whereas r-ADC remained higher than normal in affected portions of the bones.

Conclusion: Diffusion-weighted imaging is a fast, sensitive magnetic resonance imaging sequence in detection of active sacroiliitis. It does not require contrast agent and can be safely used as an adjunct to conventional magnetic resonance images. $r-A D C$ is also highly sensitive in detecting active sacroiliitis and may be used as an alternative to standard ADC measurements for the demonstration of inflammation. It helps eliminate individual bone marrow differences by using patients' own normal bone marrow measurements and increases diagnostic accuracy.

Keywords: Apparent diffusion coefficient; diffusion-weighted imaging; magnetic resonance imaging; sacroiliitis.
\end{abstract}

Diffusion-weighted magnetic resonance imaging is predicated on the basis of the fact that any particle in a liquid (e.g. water) that is stimulated by thermal energy is randomly displaced causing Brownian motion of the particles also named as molecular diffusion creating signal alteration in tissues. ${ }^{1}$ Intravoxel incoherent motion of water molecules in the extracellular space is measured from diffusion-weighted imaging (DWI) with at least two identified $b$ values by combining the effects of water diffusion and capillary perfusion. The term apparent diffusion coefficient (ADC) is a quantitative parameter calculated from diffusion-weighted images. ${ }^{2}$ Various pathological conditions cause alteration of the mobility of water molecules in biological

Received: January 20, 2016 Accepted: February 21, 2016 Published online: April 20, 2016

Correspondence: İclal Erdem Toslak, MD. Antalya Eğitim ve Araştırma Hastanesi Radyoloji Kliniği, 07100 Antalya, Turkey.

Tel: +90 242 - 2494990 e-mail: driclalerdem@yahoo.com

(02016 Turkish League Against Rheumatism. All rights reserved. 
tissues and can be detectable with DWI. ${ }^{3}$ DWI is most commonly used in the assessment of acute stroke. ${ }^{3}$ Other clinical applications include demyelinating diseases (e.g. multiple sclerosis), brain tumors and abscess, and musculoskeletal abnormalities. ${ }^{3,4}$ Musculoskeletal abnormalities assessed by DWI are muscle, cartilage and soft tissue diseases, several spinal abnormalities, and bone marrow edema. ${ }^{4}$ Acute osteoporotic and metastatic fractures of vertebra, spinal infection and malignancy can be discriminated by the help of DWI. Detection of skull metastasis and traumatic bone marrow edema are also possible with DWI. 3,4

Seronegative spondyloarthropathies ( $\mathrm{SpA}$ ) are a group of diseases composed of ankylosing spondylitis (AS), Reiter syndrome, enteropathic arthritis, psoriatic arthritis, and undifferentiated SpA. ${ }^{5}$ There is no relationship between the rheumatoid factor and the disease group, and the presence of class I human histocompatibility leukocyte antigen-B27 has been shown to increase 20 times the risk of developing a SpA as compared to general population. ${ }^{6,7}$ Sacroiliac joints are primarily affected in SpAs and sacroiliitis is an important diagnostic criterion in the disease group. ${ }^{4,6}$ Inflammatory back pain is the primary symptom of sacroiliitis that is aggravated by rest and improved by physical activity. ${ }^{8}$ Because of difficulty in differentiating mechanical back pain and inflammatory back pain, imaging is important in the diagnosis of sacroiliitis. ${ }^{6}$ Although the first step imaging modality is radiography, it may take two to five years to become apparent on plain films that retard the diagnosis. Therefore, further imaging modalities are needed to diagnose the entity earlier. ${ }^{4}$ The ability of earlier diagnosis with advanced magnetic resonance imaging (MRI) techniques like dynamic contrast-enhanced MRI or DWI even in the lack of positive laboratory findings for SpA make the method accessible as compared to plain radiographs or computed tomography especially in the active stage of disease. ${ }^{4,6}$

The diffusivity of an individual tissue may be affected by many in vivo (like patients' age and body temperature) and ex vivo (like different MRI devices, area of region of interest [ROI], $b$ value) factors. To eliminate these variations, use of normalized ADC, also named as relative ADC $(\mathrm{r}-\mathrm{ADC})$ is suggested which is calculated by the formula ADC lesion/ADC reference site. ${ }^{9}$ The relativity of the ADC measurements is supposed to be minimized with the help of $\mathrm{r}$-ADC, which are calculated by dividing the lesion $\mathrm{ADC}$ values by the ADC values of the reference organ. ${ }^{10}$

In this study, we aimed to evaluate the role of DWI in detection of active sacroiliitis and compare the ADC and $\mathrm{r}$-ADC values by using vertebra and iliac wings as reference organs.

\section{PATIENTS AND METHODS}

This study was conducted between September 2013 and January 2015 in Antalya Education and Research Hospital Hospital. The study included 56 patients (26 males, 30 females; mean age $37.7 \pm 10.1$ years; range 18 to 66 years) with chronic back pain (symptoms $>3$ months) and without history of sacroiliitis who underwent MRI. The patients had not been diagnosed as SpA before. The study was approved by Antalya Education and Research Hospital Institutional Review Board. Written informed consent was obtained from all subjects. The study was conducted in accordance with the principles of the Declaration of Helsinki.

We used the Berlin algorithm for the diagnosis of axial SpA and calculating the percentage of the probability of SpA. According to Berlin algorithm, assessment of inflammatory back pain might lead to the evaluation of other clinical features including positive family history, positive response to non-steroidal anti-inflammatory drugs, acute phase reactants (raised erythrocyte sedimentation rate/C-reactive protein), presence of dactylitis, uveitis, psoriasis, alternating buttock pain, asymmetrical arthritis, heel pain, and Crohn's disease which is followed by histocompatibility leukocyte antigen-B27 testing and skeletal imaging. ${ }^{11}$ Depending on the presence or absence of the above mentioned features of SpA, the probability of the presence of axial SpA was determined. A probability of $\geq 90 \%$ was used to make a diagnosis of axial SpA. ${ }^{11}$ Exclusion criteria were pregnancy, being younger than 18 years old, current infections, malignity, known metabolic diseases, usual contraindications for MRI (i.e. cardiac pacemakers, neurostimulators, ferromagnetic implants, etc.), and known allergy to gadolinium-based contrast agents. 
All patients underwent MRI at 1.5-Tesla superconducting eight channel MRI system (Achieva, Philips Medical Systems, Best, The Netherlands) provided with high-speed gradients. The following sequences of sacroiliac joints in the oblique coronal and axial planes as the standard protocol were obtained in the supine positions with a high-resolution phased-array coil: turbo spin-echo $\mathrm{T}_{1}$-weighted (repetition time/echo time, 600/minimum), turbo spin-echo $\mathrm{T}_{2}$-weighted (repetition time/echo time, 2100/80), $\mathrm{T}_{2}$-weighted spectral presaturation with inversion recovery (SPIR) (3100/70), $\mathrm{T}_{1}$-weighted SPIR (600/ minimum) with and without gadolinium-based contrast agents $(0.1 \mathrm{mmoL} / \mathrm{kg}$ of body weight). All sequences contained lower lumbar spine (Figures 1a-c).
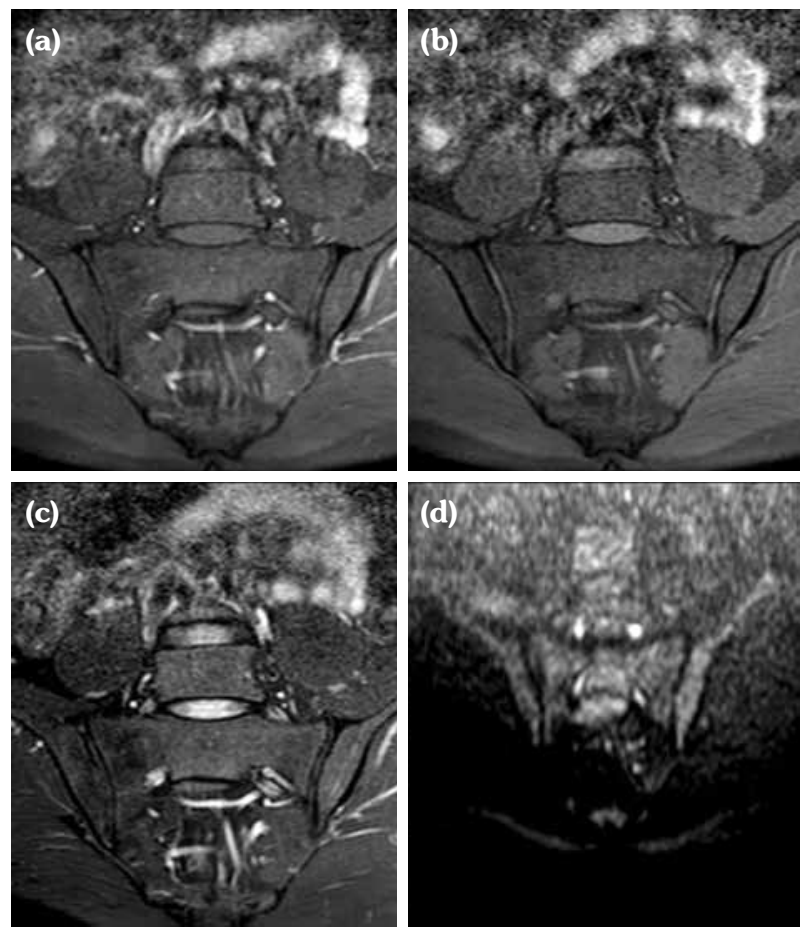

Figure 1. Thirty-seven-year-old male patient with chronic low back pain of mechanical origin. (a) Coronal oblique $\mathrm{T}_{2}$-weighted spectral presaturation with inversion recovery. (b) $\mathrm{T}_{1}$-weighted spectral presaturation with inversion recovery. (c) Postcontrast $\mathrm{T}_{1}$-weighted spectral presaturation with inversion recovery images show that there was no signal abnormality in sacroiliac joints and subarticular areas. (d) On coronal oblique diffusionweighted images at b value of $600 \mathrm{~s} / \mathrm{mm}^{2}$, sacroiliac joints and subarticular areas were also homogenous and normal appearing.
Diffusion-weighted imaging was performed by single-shot spin-echo echo-planar imaging technique with diffusion gradient $b$ values of 0 and $600 \mathrm{~s} / \mathrm{mm}^{2}$ (Figure 1d). The following DWI parameters were used: field of view: 25 (right-left) x 32 (anteroposterior) cm; number of excitations: 2; matrix size: $176 \times 176$; slice thickness: $4 \mathrm{~mm}$; repetition time/echo time, 8000/minimum; intersection gap: none.

Sacroiliac joints were assessed according to ankylosing spondylitis criteria. Before evaluation of standard MRI protocol, patients' information was removed from images. Afterwards, two experienced radiologists evaluated the images for the presence of inflammatory activity twice at separate sessions to assess interobserver variability. Following the examination of $\mathrm{T}_{2}$-weighted SPIR, contrast-

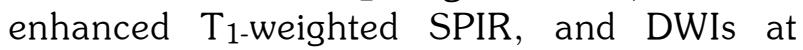
$\mathrm{b}$ values of $600 \mathrm{~s} / \mathrm{mm}^{2}$, two radiologists and rheumatologists reached a consensus on the disease activity in the light of positive features of Berlin criteria. The DWIs were transferred to a separate workstation (Phillips, Extended MR workspace, 2.6.3.2.HF3, Netherlands). ADC maps were generated. $T_{2}$-weighted SPIR images, contrast-enhanced images and diffusion-weighted images with ADC maps were put on the screen side by side. In the disease group, the hyperintense lesions on $\mathrm{T}_{2}$-weighted SPIR images and enhanced focuses on contrastenhanced images were noted and the same lesions were marked on DWI images and ADC map. A circular ROI with a range of $40-75 \mathrm{~mm}^{2}$ was placed in those areas. In the control group a circular ROI was placed in the subarticular area of joints. Also, the same process was performed for the normal-appearing bone marrow areas near the joints of the disease group (Figure 2). Four standard measurements were conducted from each joint, including two on sacral and the other two on the iliac side for all patients (Figure 3). Additionally, two measurements were conducted from the fifth lumbar vertebra and one from each iliac wing (Figure 4). All measurements were performed twice. ADC values were expressed as square millimeters per second. The $\mathrm{r}$-ADC ratio was calculated by dividing $\mathrm{ADC}$ value of subchondral bone or inflammatory lesions to ADC values of the fifth lumbar vertebra and iliac wings. 

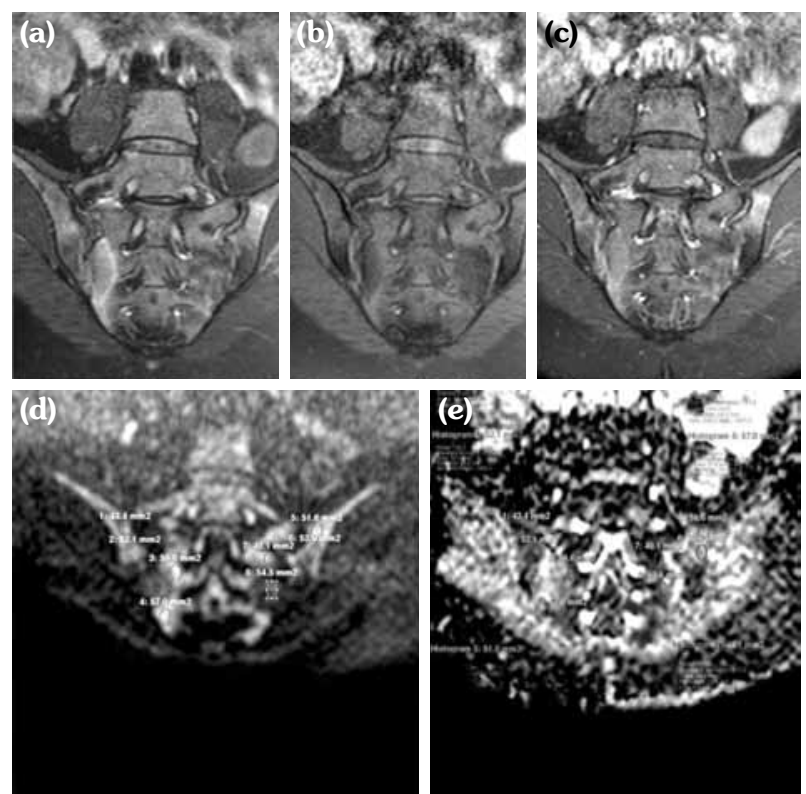

Figure 2. Thirty-four-year-old female patient with chronic low back pain of inflammatory origin. (a) Coronal oblique $\mathrm{T}_{2}$-weighted spectral presaturation with inversion recovery. (b) $\mathrm{T}_{1}$-weighted spectral presaturation with inversion recovery. (c) Postcontrast $\mathrm{T}_{1}$-weighted spectral presaturation with inversion recovery images demonstrated the subchondral bone marrow edema on both sides of joints. (d) Diffusion-weighted image lesions are hyperintense due to the restricted diffusion. (e) Circular region of interests placed on apparent diffusion coefficient map enabled to calculate the apparent diffusion coefficient values, which were higher than the normal appearing bone marrow.

\section{Statistical analysis}

Statistical calculations were performed on a personal computer using PASW statistical package for Windows version 18.0 (SPSS Inc., Chicago, IL, USA). Independent samples t tests were applied to data of normal distribution and Mann-Whitney $U$ test tests were applied to data of questionably normal distribution to compare independent groups. The distribution of categorical variables in groups was compared using Pearson Chi-square test. $P$ values lower than 0.05 were considered as statistically significant. The kappa test was used to evaluate interobserver reliability.

Contrast-enhanced $\mathrm{T}_{1}$-weighted SPIR images were used as reference standard for the presence of active inflammatory changes. Receiver operator characteristic curves were formed for each magnetic resonance sequences, and area under
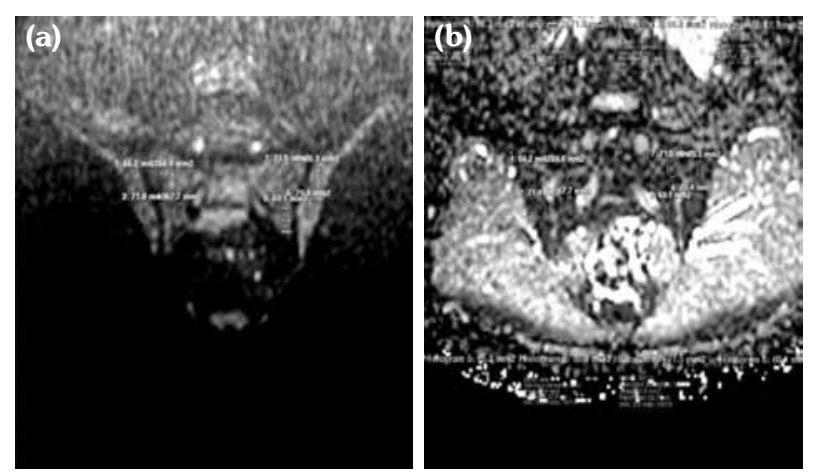

Figure 3. Thirty-seven-year-old male patient with chronic low back pain of mechanical origin. Sacroiliac joints and subarticular bony structures were normal. (a) Diffusionweighted image. (b) Four apparent diffusion coefficient values were obtained from subarticular surface of sacroiliac joints.

curve measurements were performed to assess ability of the test to discriminate disease. The area under curve values near to 1.0 represented perfect test results, while values less than or equal to 0.5 were equivalent or worse results than expected by random chance. The area under curve values were interpreted as follows: 0.9 to $0.99=$ excellent, 0.8 to $0.89=$ good, 0.7 to $0.79=$ fair or reasonable, and 0.51 to $0.69=$ poor.

\section{RESULTS}

Disease and control groups were homogenous. There was no difference in the age and the sex distribution statistically (Table 1). Thirty-nine patients with chronic back pain were diagnosed
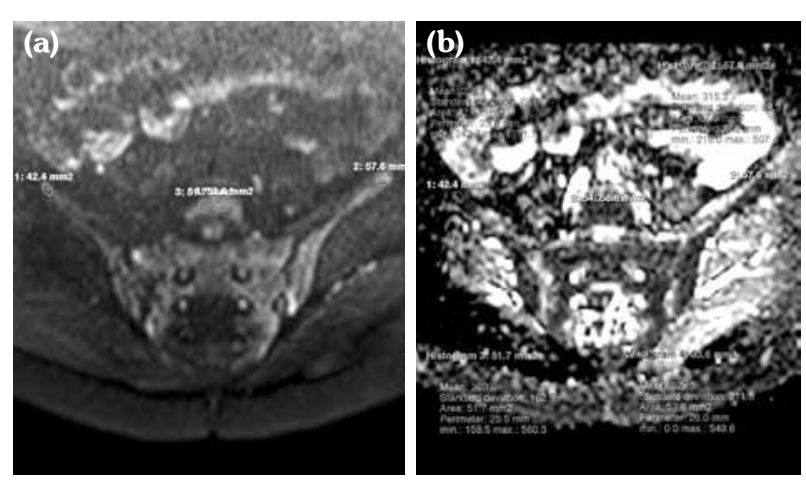

Figure 4. Fifty-two-year-old female patient presented with inflammatory back pain. (a) Diffusion-weighted image. (b) Reference apparent diffusion coefficient values were obtained from $\mathrm{L}_{5}$ vertebra and both sides of iliac wings. 
as axial spondyloarthritis with sacroiliitis and had active inflammatory changes on MRI of sacroiliac joints. Seventeen patients were diagnosed as having chronic back pain of mechanical origin. Among 39 patients with active sacroiliitis, 23 had erosions on either sacral or iliac surfaces; eight patients had sclerosis either on the right sacral bone (RSB), left sacral bone (LSB), right subchondral iliac bone (RIB) or left subchondral iliac bone (LIB) detected by plain radiographs; and sixteen patients had periarticular fat deposits in the bone marrow. Three of all patients had ankylosis while 15 with active sacroiliitis had no structural changes on MRI.

In the inflammatory back pain group, mean ADC measurements of all sacral and iliac bones were significantly higher than that of patients with mechanic back pain (Table 1). There was no significant difference between the mean ADC values of the bone marrow of L5 vertebra and iliac wings in patients with active sacroiliitis and mechanic back pain (Table 1). The mean ADC measurements of active lesions for both sides of joints for iliac and the sacral bones were significantly higher than either normalappearing bone marrow areas in patients with active sacroiliitis or in patients with mechanical back pain (Table 1).

When the r-ADC ratios of both groups' normalappearing bone marrow and inflammatory lesions calculated by fifth lumbal vertebra were compared, $r-A D C$ values of active lesions were considerably higher than normal-appearing bone marrow areas both in patients with active sacroiliitis or in patients with mechanical back pain. There was no significant difference between the $\mathrm{r}$-ADC values of both groups' normal-appearing subchondral bones calculated with $\mathrm{L}_{5}$ vertebra chosen as the reference organ (Table 1 ).

When the r-ADC ratios of both groups and inflammatory lesions calculated by iliac wing were compared, $\mathrm{r}$-ADC values of active lesions were considerably higher than normal-appearing bone marrow areas both in patients with active sacroiliitis or in patients with mechanical back pain. Except from right iliac bone, there was no significant difference between the $\mathrm{r}-\mathrm{ADC}$ values of both groups' normal-appearing iliac and sacral

Table 1. Mean apparent diffusion coefficient and $r$-apparent diffusion coefficient values between groups

\begin{tabular}{|c|c|c|c|c|c|c|c|c|c|c|c|}
\hline & \multicolumn{5}{|c|}{ Mechanic back pain } & \multicolumn{5}{|c|}{ Sacroiliitis } & \multirow[b]{2}{*}{$p$} \\
\hline & $\mathrm{n}$ & $\%$ & Mean \pm SD & Median & Min.-Max. & $\mathrm{n}$ & $\%$ & Mean \pm SD & Median & Min.-Max. & \\
\hline Age (years) & & & $39.2 \pm 9.5$ & 37 & $24-56$ & & & $36.2 \pm 10.7$ & 34 & $18-66$ & 0.239 \\
\hline Gender & & & & & & & & & & & 0.303 \\
\hline Male & 7 & 36.8 & & & & 19 & 5.1 & & & & \\
\hline Female & 12 & 63.2 & & & & 18 & 4.8 & & & & \\
\hline Vertebra & & & $392.0 \pm 110.5$ & 409 & $158-631$ & & & $433.8 \pm 104.2$ & 445 & $145-631$ & 0.126 \\
\hline Iliac wing & & & $446.9 \pm 112.6$ & 459 & $242-651$ & & & $502.3 \pm 112.3$ & 519 & $262-719$ & 0.100 \\
\hline Right iliac bone & & & $415.2 \pm 67.9$ & 429 & $314-536$ & & & $709.0 \pm 255.5$ & 650 & 291-1399 & 0.001 \\
\hline RSB & & & $464.3 \pm 94.5$ & 448 & $312-679$ & & & $586.3 \pm 176.6$ & 532 & $299-1211$ & 0.003 \\
\hline LIB & & & $507.8 \pm 95.0$ & 518 & $319-698$ & & & $675.0 \pm 261.4$ & 590 & $343-1328$ & 0.021 \\
\hline LSB & & & $497.7 \pm 81.7$ & 492 & $319-630$ & & & $606.9 \pm 183.6$ & 562 & $311-1122$ & 0.027 \\
\hline Lesion right iliac bone & & & $415.2 \pm 67.9$ & 429 & $314-536$ & & & $1182.8 \pm 277.4$ & 1148 & $699-1884$ & 0.001 \\
\hline Lesion RSB & & & $464.3 \pm 94.5$ & 448 & $312-679$ & & & $1175.5 \pm 281.3$ & 1187 & $524-1639$ & 0.001 \\
\hline Lesion LIB & & & $507.8 \pm 95.0$ & 518 & $319-698$ & & & $1243.2 \pm 277.9$ & 1230 & $631-1725$ & 0.001 \\
\hline Lesion LSB & & & $497.7 \pm 81.7$ & 492 & $319-630$ & & & $1100.8 \pm 307.4$ & 1128 & $581-1710$ & 0.001 \\
\hline Right iliac bone/vertebra & & & $1.2 \pm 0.5$ & 1.1 & $0.7-2.5$ & & & $1.7 \pm 1.4$ & 1.4 & $0.7-9.0$ & 0.053 \\
\hline $\mathrm{RSB} /$ vertebra & & & $1.3 \pm 0.4$ & 1.2 & $0.8-2.6$ & & & $1.5 \pm 1.2$ & 1.3 & $0.8-8.4$ & 0.284 \\
\hline LIB/vertebra & & & $1.4 \pm 0.5$ & 1 & $0.7-2$ & & & $1.7 \pm 1.2$ & 2 & $0.7-8$ & 0.205 \\
\hline LSB/vertebra & & & $1.4 \pm 0.5$ & 1.2 & $0.7-2.5$ & & & $1.5 \pm 0.8$ & 1.3 & $0.8-5.9$ & 0.658 \\
\hline Lesion RSB/vertebra & & & $1.3 \pm 0.4$ & 1.2 & $0.8-2.6$ & & & $3.2 \pm 1.7$ & 2.9 & $0.9-8.3$ & 0.001 \\
\hline Lesion LIB/vertebra & & & $1.4 \pm 0.5$ & 1 & $0.7-2$ & & & $3.2 \pm 1.9$ & 2.8 & 1.3-11.9 & 0.001 \\
\hline Lesion LSB/vertebra & & & $1.4 \pm 0.5$ & 1.2 & $0.7-2.5$ & & & $2.6 \pm 0.8$ & 2.7 & $1.2-5.2$ & 0.001 \\
\hline Right iliac bone/iliac wing & & & $1.0 \pm 0.3$ & 0.9 & $0.5-2.1$ & & & $1.5 \pm 0.5$ & 1.4 & $0.4-2.6$ & 0.001 \\
\hline RSB/iliac wing & & & $1.1 \pm 0.3$ & 1.0 & $0.6-1.7$ & & & $1.2 \pm 0.4$ & 1.1 & $0.4-2.3$ & 0.414 \\
\hline LIB/iliac wing & & & $1.2 \pm 0.4$ & 1 & $0.6-2$ & & & $1.4 \pm 0.5$ & 1 & $0.6-3$ & 0.205 \\
\hline LSB/iliac wing & & & $1.2 \pm 0.3$ & 1.1 & $0.8-1.9$ & & & $1.3 \pm 0.5$ & 1.2 & $0.5-2.4$ & 0.509 \\
\hline Lesion right iliac bone/iliac wing & & & $1.0 \pm 0.3$ & 0.9 & $0.5-2.1$ & & & $2.5 \pm 1.0$ & 2.4 & $1.2-6.1$ & 0.001 \\
\hline Lesion RSB/iliac wing & & & $1.1 \pm 0.3$ & 1.0 & $0.6-1.7$ & & & $2.5 \pm 1.1$ & 2.3 & $1.2-5.3$ & 0.001 \\
\hline Lesion LIB/iliac wing & & & $1.2 \pm 0.4$ & 1.1 & $0.6-2$ & & & $2.5 \pm 0.8$ & 2.7 & $0.9-4.1$ & 0.001 \\
\hline Lesion LSB/iliac wing & & & $1.2 \pm 0.3$ & 1.1 & $0.8-1.9$ & & & $2.4 \pm 0.9$ & 2.2 & $1.0-4.0$ & 0.001 \\
\hline
\end{tabular}




\begin{tabular}{|c|c|c|c|}
\hline & Area under curve & $95 \% \mathrm{CI}$ & $p$ \\
\hline Lesion right iliac bone & 0.976 & 0.890-0.999 & 0.001 \\
\hline Lesion right sacral bone & 0.853 & $0.727-0.936$ & 0.001 \\
\hline Lesion left iliac bone & 0.814 & $0.681-0.938$ & 0.001 \\
\hline Lesion left sacral bone & 0.811 & $0.678-0.906$ & 0.001 \\
\hline Lesion right iliac bone/vertebra & 0.860 & $0.736-0.941$ & 0.001 \\
\hline Lesion right sacral bone/vertebra & 0.718 & $0.576-0.834$ & 0.001 \\
\hline Lesion left iliac bone/vertebra & 0.704 & $0.561-0.823$ & 0.001 \\
\hline Lesion left sacral bone/vertebra & 0.740 & $0.599-0.851$ & 0.001 \\
\hline Lesion right iliac bone/iliac wing & 0.923 & $0.815-0.979$ & 0.001 \\
\hline Lesion right sacral bone/iliac wing & 0.746 & $0.606-0.856$ & 0.001 \\
\hline Lesion left iliac bone/iliac wing & 0.649 & $0.649-0.776$ & 0.001 \\
\hline Lesion left sacral bone/iliac wing & 0.746 & $0.606-0.856$ & 0.001 \\
\hline
\end{tabular}

bones calculated with iliac wings chosen as the reference organ (Table 1).

Receiver operator characteristic curves were formed for both ADC and $\mathrm{r}$-ADC values (Table 2). $\mathrm{By}$ using the contrast-enhanced $\mathrm{T}_{1}$-weighted images as the reference standard, area under curve (AUC) values were obtained. AUC values of lesions were 0.976 for RIB, 0.814 for LIB, 0.811 for LSB, and 0.853 for RSB. AUC values of both sacral and left iliac bones were interpreted as good, whereas AUC values of right iliac bone were interpreted as excellent.

Area under curve values of lesions calculated with the reference organ chosen as $\mathrm{L}_{5}$ vertebra were 0.860 for RIB, 0.718 for RSB, 0.704 for LIB, and 0.740 for LSB. AUC values of lesions in right iliac bones were interpreted as good, whereas AUC values of lesions on both sides of sacral bones and left iliac bones were interpreted as reasonable.

Area under curve values of lesions calculated with the reference organ chosen as iliac wings were 0.923 for RIB, 0.746 for RSB, 0.649 for LIB, and 0.746 for LSB. AUC values of lesions in right iliac bone were interpreted as excellent, whereas AUC values of lesions on both sides of sacral and left iliac bones were interpreted as reasonable.

Good values of all the three methods indicate that both $\mathrm{ADC}$ and $\mathrm{r}-\mathrm{ADC}$ values regardless of the location can discriminate active lesions in sacroiliitis similar to contrast-enhanced $\mathrm{T}_{1 \text {-weighted images. }}$

When receiver operating characteristic analysis of three methods was compared, AUC values of ADC measurements without normalizing with a reference organ were the highest as compared to $r$-ADC measurements. AUC values for lesions calculated with $\mathrm{r}$-ADC with the reference organ chosen as $\mathrm{L}_{5}$ vertebra were higher than the AUC values for lesions calculated with $\mathrm{r}$-ADC with the reference organ chosen as iliac wings.

The agreement between the two radiologists was analyzed with kappa ( $\kappa)$, according to the following interpretation of the $\kappa$ value: $0.00-0.20$, poor agreement; $0.21-0.40$, fair agreement; 0.41-0.60, moderate agreement; 0.61-0.80, considerable agreement; and 0.81-1.00, nearly perfect agreement. In our study, authors compared the DWI images with the contrastenhanced $\mathrm{T}_{1}$-weighted fat saturated images in

Table 3. Interobserver reliability for $\mathrm{T}_{1}$-weighted images and diffusion-weighted images

\begin{tabular}{lcccc}
\hline Image & Agreement $\%$ & $\kappa$ & $95 \%$ CI for $\kappa$ & $p$ \\
\hline Diffusion-weighted imaging on right side & 96.5 & 0.931 & $0.837-1.026$ & $<0.001$ \\
Diffusion-weighted imaging on left side & 94.8 & 0.896 & $0.780-1.012$ & $<0.001$ \\
Contrast-enhanced T1-W SPIR and T2-SPIR on the right side & 94.8 & 0.939 & $0.874-1.004$ & $<0.001$ \\
Contrast-enhanced T 1 -W SPIR and T2-SPIR on the left side & 96.5 & 0.960 & $0.907-1.013$ & $<0.001$ \\
\hline CI: Confidence interval; SPIR: Spectral Presaturation with Inversion Recovery. & & & \\
\hline
\end{tabular}


terms of measuring variability. The percentage of agreement between the two researchers and the weighted kappa coefficients was calculated with 95\% confidence intervals which was almost perfect in detecting active sacroilitis for both contrast-enhanced $\mathrm{T}_{1}$-weighted fat saturated images and diffusion-weighted images (Table 3).

\section{DISCUSSION}

The detection of active sacroiliitis in SpA patients is critical in the management and follow-up protocol. ${ }^{6}$ Until recently, radiography was the primary imaging method to determine sacroiliitis after clinical assessment according to the modified New York criteria. ${ }^{12}$ As it may take up to 10 years for sacroiliitis to become evident on radiographs and with the progresses in the field of SpA, new diagnostic tools were needed to diagnose sacroiliitis as well as to avoid probable complications in the later stages of disease and decrease morbidity. Furthermore, with the introduction of anti-tumor necrosis factor agents having great potential to improve the outcome, earlier diagnosis of sacroiliitis became even more significant. ${ }^{12}$ MRI may depict the inflammatory changes in the sacroiliac joints years before the appearance of structural changes on the roentgenograms. ${ }^{13}$ STIR images together with the contrast-enhanced fat-suppressed $\mathrm{T}_{1}$-weighted images were used to detect inflammation. In some studies, contrastenhanced $\mathrm{T}_{1}$-weighted sequences were found to be more sensitive than STIR and fat-suppressed $\mathrm{T}_{2}$-weighted images. ${ }^{4}$

Up to date, many studies have been performed on detection of sacroiliitis with DWI. Bozgeyik et al. ${ }^{4}$ studied 42 patients with chronic low back pain (suspected spondyloarthropathy). $\mathrm{ADC}$ values of active inflammatory lesions in active sacroiliitis group were significantly higher than mechanical low back pain group. They concluded that DWI is a sensitive, fast sequence and does not require a contrast agent, which makes it a good and cost-effective alternative for imaging sacroiliac joints. DWI also allows quantifying diffusion coefficients of the lesions, which can discriminate between normal and involved subchondral bone, and offers a new alternative for follow-up. DWI may be useful in the early diagnosis and follow-up of the acute inflammatory lesions that occur in early axial spondyloarthritis. ${ }^{4}$

Gaspersic et al. ${ }^{14}$ showed in their study that ADC values of AS patients at $b$ value of 400 were higher than that of the normal bone marrow. They evaluated the effects of different therapies on enthesitis and osteitis in active AS using DWI and dynamic contrast enhanced-MRI. They concluded that quantitative MRI parameters diminished significantly with regression of the inflammatory activity. DWI and dynamic contrast enhanced-MRI were shown to be effective in quantifying changes in inflammation during the treatment of AS and may be convenient for assessing treatment efficacy. ${ }^{14}$

Ai et al. ${ }^{15}$ evaluated 52 patients of whom 16 had early active sacroiliitis. They found that ADC values of inflammatory lesions of AS patients at $b$ values of $600 \mathrm{~s} / \mathrm{mm}^{2}$ were significantly higher than that of adjacent relatively normal regions and also the latter was higher than that of low back pain and control group. They concluded that DWI was established as a highly reliable imaging tool for the early diagnosis and followup monitoring of AS patients. With the rapid progress of advanced imaging techniques, DWI and whole body-DWI will likely provide a widened application in the early diagnosis and long-term follow-up in AS patients. ${ }^{15}$

Moreover, Sanal et al. ${ }^{2,16}$ performed two studies in 2013 with 21 and 33 patients and stated that DWI is comparable to visual analysis and active lesions in sacroiliitis of AS patients serving an adjunct to STIR. Also, they demonstrated that DWI may reflect the inflammatory state of disease by quantifying the bone marrow changes. The ADC values of lesions in one of their study were significantly lower than that of lesions. Their other publication was about the visual analysis and perception of the active lesions in sacroiliitis of AS patients and they stated that DWI was comparable in visual analysis and perception. ${ }^{2,16}$

Gezmis et al. ${ }^{6}$ evaluated 62 patients diagnosed as SpA and 40 healthy controls. They found that the differences in mean ADC values between disease and control groups were statistically significant. In their study, the mean ADC value of $0.86 \times 10^{-3} \mathrm{~mm}^{2} / \mathrm{s}$ obtained from active lesions was significantly higher than the control group. Also, they showed that the mean $\mathrm{ADC}$ values of the 
normal-appearing bone marrow of patients were significantly higher than the control group. ${ }^{6}$

In their study on multiparametric MRI in the detection of early inflammatory sacroiliitis, Boy et al. ${ }^{17}$ used $b$ values of $0,100,600$ and $1000 \mathrm{~s} / \mathrm{mm}^{2}$ when acquiring diffusion-weighted images. They concluded that the addition of DWI does not provide significant value in the diagnosis of osteitis for both experienced and relatively inexperienced radiologists. ${ }^{17}$

Dewan et al. ${ }^{18}$ evaluated 15 patients with AS, 20 patients with low back pain, and 20 control subjects. They applied whole body DWI at the $\mathrm{b}$ value of $600 \mathrm{~s} / \mathrm{mm}^{2}$ to 10 clinically confirmed AS patients. According to the results of their study, the mean ADC values of iliac and sacral bones were statistically significantly higher in the AS group than that of LBP group. There was no statistically significant difference between the mean ADC values of LBP and control group. They concluded that DWI and whole body-DWI by measuring the ADC values were sensitive and fast sequences that allow differentiation between inflammatory and mechanical low back pain. ${ }^{18}$

Zhao et al. ${ }^{19,20}$ performed two studies in 2014 and 2015 with 56 and 41 patients, respectively. In their study performed in 2014, they found that ADC values were significantly different among AS active, AS chronic, and the control groups. They stated that diffusion-weighted sequences and contrast-enhanced sequences were superior to other methods of evaluating the activity in AS. In their study performed in 2015, they evaluated tissue diffusivity, perfusion fraction (f), and pseudo-diffusion coefficient values for three groups that were consisted of AS active patients, AS chronic patients, and healthy volunteers. They found that tissue diffusivity and perfusion fraction of intravoxel incoherent motion DWI in AS show a significant difference in the values of diffusion of water molecules and fractional perfusion-related volume among the three groups. ${ }^{19,20}$

Furthermore, Zhang et al. ${ }^{21}$ evaluated 16 healthy controls and 52 patients in their study. They found a statistically significant difference between ADC values of the control and AS groups. Also, in their study, there was a statistically significant difference between the control and patient groups in the right ilium $\mathrm{ADC}$ values while there were no significant differences between the two groups in the left ilium, left sacrum and right sacrum. They concluded that the ADC values of lesions were greater than those of the unaffected portions in AS patients. ${ }^{21}$

Our study revealed that ADC values of active lesions in patients with axial SpA were significantly higher than the ADC values of unaffected parts of bone marrow in patients with active sacroiliitis as well as the ADC values of bone marrow in patients with chronic back pain that were similar with the majority of the literature. There are slight differences in the ADC values in different studies that may be attributed to the different MRI device and different $\mathrm{b}$ values. Moreover, similar to the studies of Gezmis et al. ${ }^{6}$ and $\mathrm{Ai}$ et al. ${ }^{15}$ and unlike the studies of Zhang et al. ${ }^{21}$ and Sanal et al., ${ }^{16}$ the ADC values of unaffected portions of sacroiliac joints in patients with active sacroiliitis were also greater than the ADC values of bone marrow in patients with chronic back pain. This result may be due to the extensive spread of active lesions to the subchondral bone marrow area. Another reason was that although the patients in our study admitted to Rheumatology Department first time with the presenting symptoms of axial $\mathrm{SpA}$ and were thought to be in early stages of the disease, more than half of the patients had already developed structural changes, indicating that actually they were not in early stages of the disease. The patients with chronic sacroiliitis as well might have affected the measurements in such a way that the remaining normal appearing bone marrow area was very limited because of erosions and periarticular fatty deposits. Thus, neighboring active lesion areas may have affected the measurements.

Normalized ADC, known as r-ADC, which is calculated by the formula ADC lesion/ADC reference site ${ }^{9}$ is used to normalize the ADC value and eliminate the individual differences. A number of studies have been performed by using $\mathrm{r}$-ADC values for characterization of focal pancreatic lesions, ${ }^{22}$ in liver fibrosis, ${ }^{23}$ for estimating histological grade of vesical urothelial carcinoma, ${ }^{24}$ monitoring the development of brain infarction, ${ }^{25}$ and assessing benign and malignant breast lesions. ${ }^{26}$ To our knowledge, no study has been conducted by using r-ADC values performed on the musculoskeletal system so far. In our study, we used L5 vertebra or iliac wings 
as the reference organs to eliminate individual differences of bone marrow of patients as well as the differences arising from the acquisition protocol and signal analysis. The patients' own normal bone marrow was chosen as the reference standard and a proportional value was obtained for each patient. We saw that normalized ADC values were able to detect active sacroiliitis as well as the conventional DWI. r-ADC measurements of lesions could discriminate the disease with the reference site chosen as either vertebra or iliac wings. In $\mathrm{r}-\mathrm{ADC}$ values calculated with the $\mathrm{L}_{5}$ vertebra, there was no significant difference between unaffected portions of bone marrow areas in patients with sacroilitis and mechanic back pain whereas the $\mathrm{r}-\mathrm{ADC}$ remained still higher than the normal bone marrow areas in patients with sacroiliitis or mechanic back pain. So, we think that this method may be used to detect real active lesions by normalizing unaffected portions of sacroiliitis patients that were also higher in DWI without normalizing method. As a consequence, we think that $\mathrm{L}_{5}$ vertebra is a more useful reference organ in calculating $\mathrm{r}-\mathrm{ADC}$ than iliac wings. Furthermore, because of the difficulty in measuring the $\mathrm{ADC}$ values of iliac wings owing to the narrow zone to put the ROI in MRI sections, choosing the $\mathrm{L}_{5}$ vertebra as the reference organ is much easier in addition to the advantage of having the similar architecture with the bones of the sacroiliac joint. Also, AUC values for lesions calculated with $r$-ADC with the reference organ chosen as $\mathrm{L}_{5}$ vertebra were higher than the AUC values for lesions calculated with $\mathrm{r}$-ADC with the reference organ chosen as iliac wings. This method may be safely used to detect active sacroiliitis in individuals that have pathologic conditions causing generalized bone marrow edema that might affect subchondral bone marrow ADC values. By this way, quantification of active lesions can be performed more accurately. We did not have any patient with generalized bone marrow edema; however, particularly younger patients had more active bone marrow. Still, the number of those patients was not sufficient to conduct a statistical analysis to compare with the patients with less active bone marrow edema. Further studies are required with younger and older populations to compare vertebral bone marrow and $r-A D C$ values of sacroiliac joints of the groups to understand effectivity of $r-A D C$ values.
In contrast to the study of Boy et al., ${ }^{17}$ we think that addition of DWI to the standard MRI protocol of sacroiliac joints increases the awareness of active sacroiliitis and as well as the rest of the researches on DWI of sacroiliitis.

Our study has some limitations. One disadvantage of the DWI over the conventional MRI sequences was the insufficiency of DWI to show anatomic structures due to the echoplanar imaging-based DWI, which suffers from geometrical image distortions owing to the long gradient echo train lengths. ${ }^{16}$ Most of the time, a conventional sequence in the same plane was needed to demonstrate the joints on DWI. Nevertheless, sufficient contrast between the lesions and normal-appearing bone marrow could be obtained to reveal the active stage of the disease. Dietrich et al. ${ }^{27}$ and Raya et al. ${ }^{28}$ studied with a new technique called as rapid acquisition with relaxation enhancement-based DWI for musculoskeletal applications. In this technique, image distortions were less than the single-shot echo-planar imaging technique and enabled more anatomic information, as the muscle and fat signals were considerably higher. For optimization and development of the image quality, further studies are needed to employ new DWI strategies in the musculoskeletal field.

The lack of standardization in DW-MRI (including acquisition protocol and signal analysis) is an important limitation for a valid inter-study comparison. To overcome this limitation, normalized ADC might be considered as future research tracks since technological innovation continues to enhance the nature of clinical DW-MRI and more refined information handling frameworks are currently accessible.

Another limitation of our study was that, in our hospital, near all of the patients undergoing sacroiliac joint examination were referred from the rheumatology department. Thus, the radiologists knew that those patients were suffering from inflammatory back pain and they were aware of the high probability of MRI to be positive. This was a contributing factor for high sensitivity and reliability.

Another restriction of our study was its small sample size. Therefore, studies with larger populations are needed. 
In conclusion, DWI is a fast, sensitive MRI sequence in the detection of active sacroiliitis. It helps to differentiate normal and pathological subchondral bone marrow by measuring ADC values. It does not require contrast agent and may be safely used as an adjunct to conventional MRI sequences. Also, ADC normalization is a promising tool to improve the accuracy of ADC measurements and diagnostic performances. This method might be used as an alternative to standard ADC measurements for the demonstration of inflammation in detecting active sacroiliitis. It helps eliminate individual bone marrow differences by using patients' own normal bone marrow measurements and increases diagnostic accuracy.

\section{Acknowledgements}

The authors thank Professor Mehmet Ziya Firat for his assistance with the data analysis.

\section{Declaration of conflicting interests}

The authors declared no conflicts of interest with respect to the authorship and/or publication of this article.

\section{Funding}

The authors received no financial support for the research and/or authorship of this article.

\section{REFERENCES}

1. Hagmann $\mathrm{P}$, Jonasson L, Maeder $\mathrm{P}$, Thiran JP, Wedeen VJ, Meuli R. Understanding diffusion MR imaging techniques: from scalar diffusion-weighted imaging to diffusion tensor imaging and beyond. Radiographics 2006;26:205-23.

2. Sanal HT, Yilmaz S, Kalyoncu U, Cinar M, Simsek $\mathrm{I}$, Erdem $\mathrm{H}$, et al. Value of DWI in visual assessment of activity of sacroiliitis in longstanding ankylosing spondylitis patients. Skeletal Radiol 2013;42:289-93.

3. Raya JG, Dietrich O, Reiser MF, Baur-Melnyk A. Methods and applications of diffusion imaging of vertebral bone marrow. $\mathrm{J}$ Magn Reson Imaging 2006;24:1207-20.

4. Bozgeyik Z, Ozgocmen S, Kocakoc E. Role of diffusion-weighted MRI in the detection of early active sacroiliitis. AJR Am J Roentgenol 2008;191:980-6.

5. Althoff CE, Feist E, Burova E, Eshed I, Bollow M, Hamm B, et al. Magnetic resonance imaging of active sacroiliitis: do we really need gadolinium? Eur J Radiol 2009;71:232-6.

6. Gezmis E, Donmez FY, Agildere M. Diagnosis of early sacroiliitis in seronegative spondyloarthropathies by DWI and correlation of clinical and laboratory findings with ADC values. Eur J Radiol 2013;82:2316-21.

7. Braun J, Bollow M, Remlinger G, Eggens U, Rudwaleit $\mathrm{M}$, Distler A, et al. Prevalence of spondylarthropathies in HLA-B27 positive and negative blood donors. Arthritis Rheum 1998;41:58-67.

8. Paparo F, Revelli M, Semprini A, Camellino D, Garlaschi A, Cimmino MA, et al. Seronegative spondyloarthropathies: what radiologists should know. Radiol Med 2014;119:156-63.

9. Park SO, Kim JK, Kim KA, Park BW, Kim N, Cho $G$, et al. Relative apparent diffusion coefficient: determination of reference site and validation of benefit for detecting metastatic lymph nodes in uterine cervical cancer. J Magn Reson Imaging 2009;29:383-90.

10. Sivrioğlu AK, Özcan Ü, Türk A, Ulus S, Ylldız ME, Sönmez G, et al. Evaluation of the placenta with relative apparent diffusion coefficient and T2 signal intensity analysis. Diagn Interv Radiol 2013;19:495500.

11. Rudwaleit M, van der Heijde D, Khan MA, Braun J, Sieper J. How to diagnose axial spondyloarthritis early. Ann Rheum Dis 2004;63:535-43.

12. Maksymowych WP, Weber U. Diagnostic utility of MRI in early spondyloarthritis. Curr Rheumatol Rep 2011;13:402-8.

13. Raychaudhuri SP, Deodhar A. The classification and diagnostic criteria of ankylosing spondylitis. J Autoimmun 2014;48-49:128-33.

14. Gaspersic N, Sersa I, Jevtic V, Tomsic M, Praprotnik S. Monitoring ankylosing spondylitis therapy by dynamic contrast-enhanced and diffusion-weighted magnetic resonance imaging. Skeletal Radiol 2008;37:123-31.

15. Ai F, Ai T, Li X, Hu D, Zhang W, Morelli JN. Value of diffusion-weighted magnetic resonance imaging in early diagnosis of ankylosing spondylitis. Rheumatol Int 2012;32:4005-13.

16. Sanal HT, Yilmaz S, Simsek I, Cinar M, Erdem H, Pay $\mathrm{S}$, et al. Apparent diffusion coefficients of sacroiliitis in patients with established ankylosing spondylitis. Clin Imaging 2013;37:734-9.

17. Boy FN, Kayhan A, Karakas HM, Unlu-Ozkan F, Silte D, Aktas I. The role of multi-parametric MR imaging in the detection of early inflammatory sacroiliitis according to ASAS criteria. Eur J Radiol 2014;83:989-96.

18. Dewan K, El-saadany H. Diagnostic value of diffusion weighted magnetic resonance image in early ankylosing spondylitis. Egypt $\mathrm{J}$ Radiol Nucl Med. Elsevier B.V 2014;45:1-6.

19. Zhao YH, Li SL, Liu ZY, Chen X, Zhao XC, $\mathrm{Hu}$ SY, et al. Detection of Active Sacroiliitis with Ankylosing Spondylitis through Intravoxel Incoherent Motion Diffusion-Weighted MR Imaging. Eur Radiol 2015;25:2754-63.

20. Zhao Y, Han X, Hu S, Shi X, Zhao J, Sun E, et al. The relevance of ADC value, T1intensive rate and the clinical activities in ankylosing spondylitis 
sacroiliitis. Zhonghua Yi Xue Za Zhi 2014;94:27503. [Abstract]

21. Zhang P, Yu K, Guo R, Shah S, Morelli JN, Runge V a., et al. Ankylosing spondylitis: correlations between clinical and MRI indices of sacroiliitis activity. Clin Radiol 2015;70:62-6.

22. Barral M, Sebbag-Sfez D, Hoeffel C, Chaput U, Dohan A, Eveno C, et al. Characterization of focal pancreatic lesions using normalized apparent diffusion coefficient at 1.5-Tesla: preliminary experience. Diagn Interv Imaging 2013;94:619-27.

23. El-Hariri M, Ali TFT, Hussien HIM. Apparent diffusion coefficient (ADC) in liver fibrosis: Usefulness of normalized ADC using the spleen as reference organ. Egypt J Radiol Nucl Med 2013;44:441-51.

24. Wang HJ, Pui MH, Guo Y, Li SR, Liu MJ, Guan J, et al. Value of normalized apparent diffusion coefficient for estimating histological grade of vesical urothelial carcinoma. Clin Radiol 2014;69:727-31.

25. Shen J-M, Xia X-W, Kang W-G, Yuan J-J, Sheng L. The use of MRI apparent diffusion coefficient (ADC) in monitoring the development of brain infarction. BMC Med Imaging 2011;11:2.

26. Jiang XY, Xie QZ, Cao XS, Gao T, Xing CY, Li XL. Value of diffusion weighted imaging in the differential diagnosis of benign and malignant breast lesions at 3.0T MRI. Genet Mol Res 2014;13:7773-9.

27. Dietrich O, Raya JG, Sommer J, Deimling M, Reiser MF, Baur-Melnyk A. A comparative evaluation of a RARE-based single-shot pulse sequence for diffusionweighted MRI of musculoskeletal soft-tissue tumors. Eur Radiol 2005;15:772-83.

28. Raya JG, Dietrich O, Birkenmaier C, Sommer J, Reiser MF, Baur-Melnyk A. Feasibility of a RAREbased sequence for quantitative diffusion-weighted MRI of the spine. Eur Radiol 2007;17:2872-9. 